\title{
Bacterial age distribution in soil - life cycles in hot and cold spots
}

\author{
Benedict Borer ${ }^{1}$ and Dani Or ${ }^{1}$ \\ ${ }^{1}$ ETH Zürich
}

September 24, 2020

\begin{abstract}
Soil bacteria live in patchy and dynamic environments where cells in adjacent microhabitats may realize vastly different generation times ranging from hours to years within small soil volumes. This study links bacterial population demographics with soil conditions to better estimate mean bacterial cell ages by tracking individual lineages over space and time using a mechanistic model of bacterial life in soil. Results show heavy-tailed distributions of generation times that follow a power law across all hydration conditions, implying no simple definition of mean soil bacterial age where soil volumes may harbor cells with very broad range of ages living side by side. The study highlights ubiquitous conditions that support a "genetic reservoir" of physiological traits for each bacterial species that may be preserved in soil cold spots and reintroduced during episodic reunification events (soil wetting).
\end{abstract}

\section{Introduction}

Notwithstanding the harsh and dynamic environmental conditions, soil microbial life thrives at all scales with a single gram of fertile soil containing up to $10^{10}$ prokaryotic cells ${ }^{1}$. Remarkably, even with such high potential abundance, soil bacteria inhabit less than $1 \%$ of the available soil surface ${ }^{2}$, being largely associated with small soil volumes of nutrient-rich and densely populated hotspots (the rhizosphere, detritusphere, or within soil aggregates ${ }^{3}$ ). Aqueous phase facilitated cell dispersion, accessibility and diffusion of nutrients within soil hotspots with high cell growth rates (similar to the growth of copiotrophic bacteria grown in laboratory settings ${ }^{4}$ ). In contrast, the remaining $99 \%$ of soil surfaces (and volumes) support very little to no bacterial activity (hence the term biological "cold spots") due to a lack of nutrients ${ }^{5}$ and unfavorable hydration conditions ${ }^{6}$. These contrasting conditions may occur within small soil volumes and result in significant generation time disparity, with rapid cell proliferation coexisting next to nearly dormant bacterial cells that support their maintenance with limited prospects for growth and cell division. Even after episodic wetting events that reconnect bacterial habitats and permit temporary infusion of nutrients, subsequent internal drainage fragments the aqueous phase and growth rates drop following diminishing accessibility and availability of nutrients. We seek to understand the consequences of this common disparity in local bacterial growth rates in soil (in both space and time) ${ }^{3}$ and impacts on cell lineage propagation and average bacterial cell ages in soil under different environmental conditions (characterized by soil type, cover, climatic conditions and land use).

At present, there are no direct methods for inferring bacterial cell age within a natural soil sample. We define cell age as the elapsed time since last cell division, and generation time as the cell age at division (also known as the interdivision time, doubling time or cell-cycle time ${ }^{7}$ ). Experimentally, cell age and related generation time distributions are deduced indirectly from growth rates or microbial activity data. Multiple techniques have been used for measuring growth rates in situ, such as direct cell counts ${ }^{8}$, radioactively labeled thymidine/leucine incorporation rates $^{9,10}$ or using observed and expected mutation accumulation rates $^{11}$. More recently, omics-based methods, such as quantitative stable isotope probing (qSIP) using 
$\mathrm{H}_{2} \mathrm{O}^{18}$, have emerged as a novel technique to measure growth rates in environmental samples ${ }^{12}$ and have even enabled the quantification of growth rates at the taxon level ${ }^{13}$. Using these techniques, mean generation times of soil microbial communities have been determined for a wide range of conditions (Fig. 1).

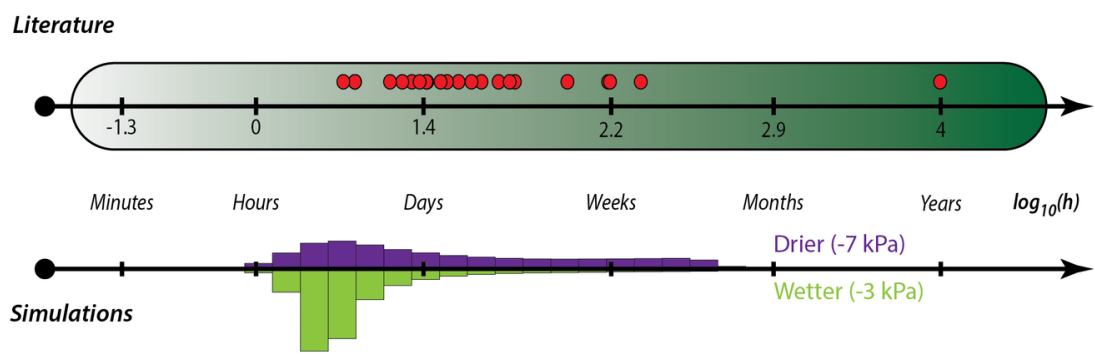

Figure 1: Distribution of experimentally measured bacterial generation times in topsoil and the rhizosphere compared to computationally obtained, generation time distributions in wet and dry conditions. Red data points represent literature values ${ }^{4,14-21}$ and represent a mean value for each sample whereas computational results show the distribution of individual generation times within one hypothetical sample under different hydration conditions.

A critical drawback of the above-mentioned techniques is that the estimated growth rates in soil are sample mean values but cannot resolve the within sample age distribution ${ }^{9}$. Additionally, most methods suffer from poorly resolved detection limits that favor identification of rapid growth, thereby biasing the demographic picture in favor of younger cells. Theoretically, we expect bacterial life in patchy soil microenvironments to produce far broader age distributions than could be resolved by current measurement methods. While direct experimental evidence from soil is scarce, we can gain insights from analogous patchy environments such as bacterial age in the phyllosphere ${ }^{22}$. Studies have tracked the reproductive success (the total number of offspring produced by an individual) of isogenic bacterial cells by linking these to leaf patchy nutrient distribution and overall local carrying capacity ${ }^{22}$. These experiments have shown a broad distribution of reproductive success (and thus generation times) for individual cell lineages despite their isogenic characteristics, which supports the role of contrasting growth conditions in soil. We hypothesize that microscale spatial variations in soil hydration state or nutrient distributions would shape the age and generation time distribution of bacterial cells. To test this hypothesis, we employ a modeling framework that combines the salient features of soil aqueous phase configurations with individual-based bacterial cell growth and dispersal ${ }^{23}$. The model tracks the life history of each bacterial cell within the simulation domain, and its lineage with reference to the localized nutrient concentrations experienced (see Fig. 2 for an example). This enables attribution of the (simulated) life history of each cell to local conditions and to its age and generation time, thus providing estimates of community age and generation time distributions by integrating across the bacterial population. The soil-like landscape and the aqueous phase distribution within it vary with hydration state giving rise to limited diffusive fluxes imposed by thin water films that support survival of bacterial cells near their maintenance rate whilst neighboring cells close to nutrient sources may proliferate at near maximum growth rates (Fig. 2). In addition to mechanistically modeling the emergence of bacterial age distribution in a soil-like system, we capitalize on a heuristic analytical formulation ${ }^{24}$ for linking local heterogeneity in bacterial growth conditions (nutrients and hydration) to the resulting bacterial age distribution.

\section{Methods: Modeling bacterial age and cell lineages in soil}

The spatially explicit mechanistic modeling framework (IndiMeSH) was used to simulate bacterial life in heterogeneous soil microhabitats ${ }^{23}$ mimicking a soil aggregate (or a hotspot around an active root segment, Fig. 2). The domain is comprised of an angular pore network occupying a spherical soil volume $(10 \mathrm{~mm}$ radius) containing individual pore channel segments with lengths of 100 microns (106,901 pores). The pore 
channels with triangular cross sections are drawn from a uniform distribution of central angles between $30^{\circ}$ and $150^{\circ}$ with inscribed pore radii sampled from a lognormal distribution with mean 30 microns and variance 10 microns. These ingredients allow for systematic variations of the domain heterogeneity by varying pore sizes in 50 randomly located regions of the pore network. Hydration conditions are prescribed as boundary conditions (for the soil volume) that translate into a distribution of micro-aqueous habitats based on the pore network (see Borer et al. $2019^{23}$ ). The effective water film thickness is derived for each individual pore channel based on its geometry and the prescribed matric potential. A carbon source was placed at the center of the simulation domain (constant concentration of $0.1 \mathrm{mM}$ ) with oxygen sources at the periphery of the pore network, following Henry's law (constant source of $0.27 \mathrm{mM}$ ). This arrangement of boundary conditions gave rise to counter gradients of oxygen and carbon mimicking conditions frequently found in natural soil hotspots ${ }^{3}$. For simplicity, we modeled bacterial cells as obligate aerobes, with Monod kinetics including carbon and oxygen limitation terms. Under optimal conditions (no oxygen or carbon limitation), the simulated bacterial cells have a mean generation time of 28 minutes. We focus on aerobic growth since this is the most common state in most near surface soils. In some soils and under certain conditions, saturated conditions throughout the soil profile may prevail, however, for simplicity and considering the long time horizons of the analyses, we neglected these cases. All model parameters concerning bacterial growth are shown in Table S1 and are based on Borer et al. $2018^{25}$. Bacterial cells are inoculated homogeneously across the domain $(1000$ cells $)$ with a total simulation time of 30 days at 10 s time steps. The age of each individual bacterial cell is captured as the time since last division. For each dividing cell, its current age is stored as the generation time while resetting the age of the daughter cells. A unique identifier is assigned to each inoculated cell that is inherited by its progeny, enabling tracking of reproductive success, generation time distribution and cell age distribution of each lineage.

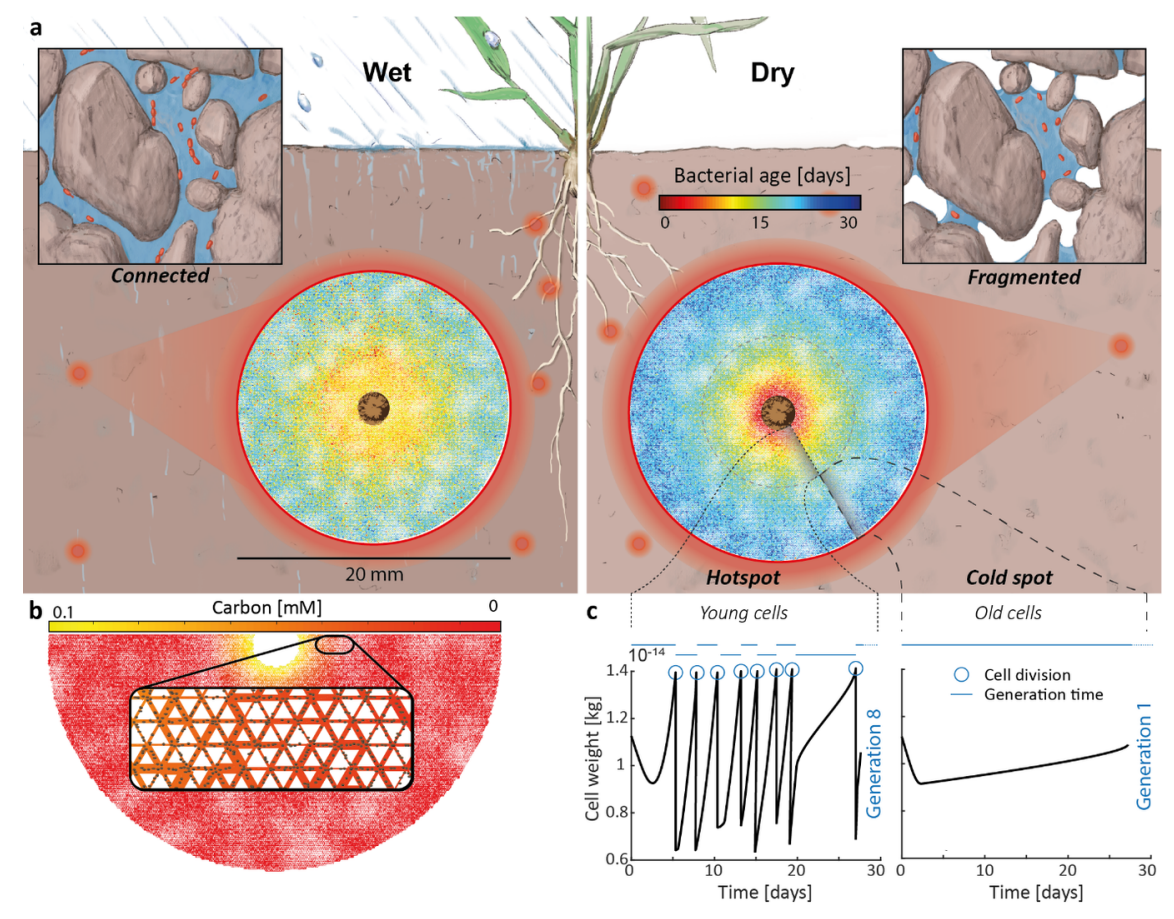

Figure 2: Bacterial population demographics shaped by diffusion and dispersal limitations around soil hot spot as affected by hydration conditions. a) Spatial visualization of simulated bacterial cell ages for $-1 \mathrm{kPa}$ and $-5 \mathrm{kPa}$ representing wet and dry conditions, respectively. Diffusion and dispersal restrictions in dry conditions expose individual lineages to harsh conditions resulting in older cells growing close to their maintenance rate. b) The model represents bacterial cells as individual agents 
inhabiting angular pore networks where aqueous diffusion is limited by thin water films, resulting in a patchy nutrient landscape and localized growth conditions and limited cell dispersal ranges. c) The spatially patchy resource landscape gives rise to bacterial "hotspots" (nutrient rich and accessible by a well-connected aqueous phase) and "cold spots" reflecting nutrient limitations and fragmentation. Cell lineages inhabiting hotspots proliferate and attain high reproductive success whereas (kin) isogenic lineages in cold spots persist with minimal prospects for growth and dispersal.

\section{Results}

Soil hydration status has been shown to be a key variable that governs multiple functions of microbial habitats such as nutrient diffusion (both aqueous and gaseous) ${ }^{6}$, community structure ${ }^{26}$, horizontal gene transfer rate $^{27}$, cell dispersal ${ }^{28-30}$ and accessibility to nutrients ${ }^{6}$. From a nutrient flux perspective, too wet or too dry soil conditions are (generally) unfavorable, due to either a lack of oxygen when saturated or limited aqueous nutrient diffusion in dry soil. Optimal nutrient and gaseous fluxes are often supported at intermediate hydration levels ${ }^{31}$. Although details of soil microhabitats are experimentally inaccessible, macroscopic hydration conditions reflect average diffusive nutrient fluxes and connectivity conditions that, in turn, affect the landscape of bacterial growth, age and generation time distributions. We use the soil water matric potential (marking the energy state of soil water) to represent prevailing hydration conditions due to its direct control over liquid organization in soil pores (via capillary interface curvatures and thickness of adsorptive films on soil surfaces). The simulations consider a simple scenario of an obligate aerobic bacterial species growing on a single carbon source (see details in the Modeling section). Fig. 3a depicts the final population size for each hydration state. For saturated conditions (matric potential of $0 \mathrm{kPa}$ and $-1 \mathrm{kPa}$ ), bacterial community growth is restricted by low oxygen diffusion through the water saturated pores limiting growth of the obligate aerobic bacteria. Intermediate hydration conditions $(-2 \mathrm{kPa}$ and $-3 \mathrm{kPa})$ create an optimal balance of aqueous and gaseous nutrient diffusion that enables rapid growth. As drier conditions set in, thin water films limit aqueous nutrient diffusion thereby reducing bacterial community size. Fig. 3b shows the empirical cumulative distribution function of the cell generation times during the simulation. On average, the shortest generation times were realized at $-2 \mathrm{kPa}$ to $-3 \mathrm{kPa}$ (optimal balance between gaseous and aqueous nutrient diffusion) and diverged towards longer mean generation times for wetter or drier conditions (Table S2). 

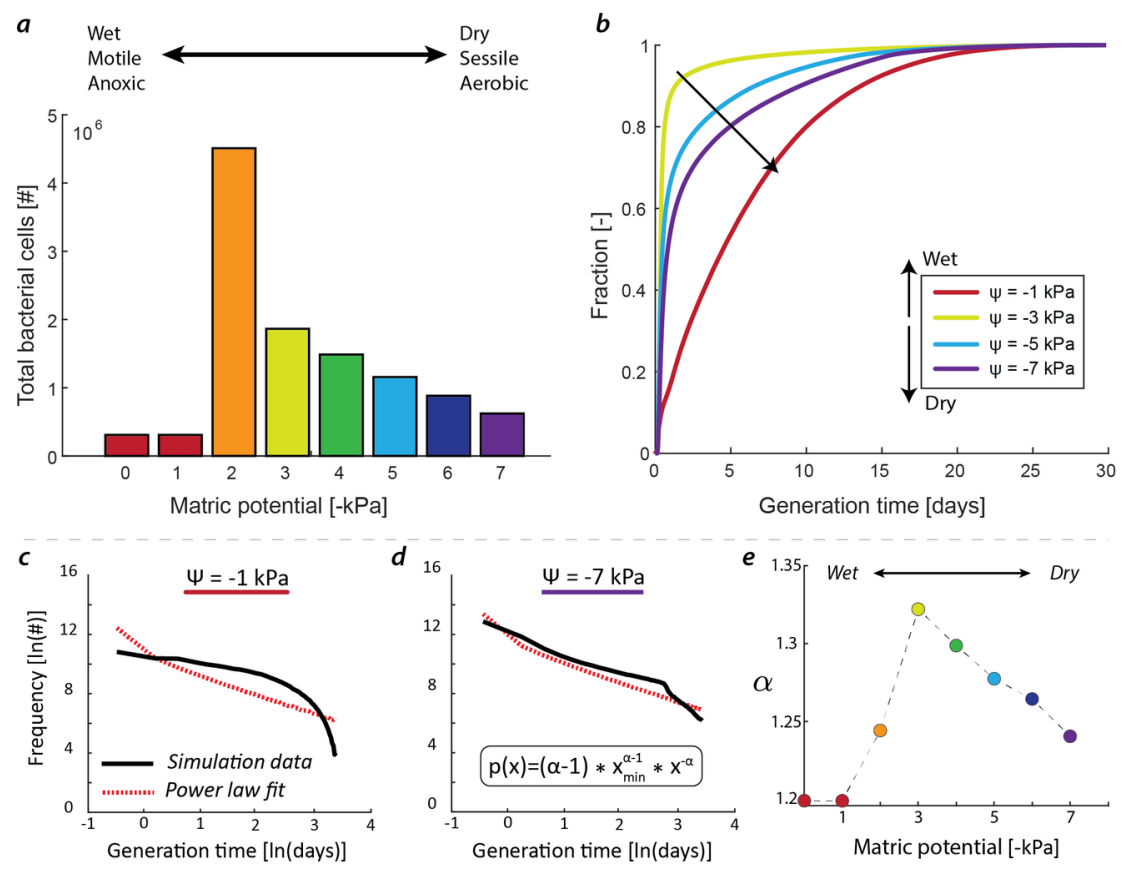

Figure 3: Variations in simulated bacterial population sizes and generation time distributions with hydration conditions . a) Final simulated population size depending on hydration conditions where optimal growth conditions occur at intermediate hydration conditions that support sufficient diffusion of both gaseous and aqueous nutrients. b) Empirical cumulative distributions of observed generation times during the simulated time. On average, the shortest generation times where obtained at -2 and $-3 \mathrm{kPa}$ due to optimal growth conditions. The cumulative density function curves for generation times at $0 \mathrm{kPa}$ and $-1 \mathrm{kPa}$ are congruent. Log-log visualization of the extrapolated simulation data and fitted power law distribution fits for the wet $(-1 \mathrm{kPa})$ scenario (c) and dry $(-7 \mathrm{kPa})$ scenario (d) where the inset in the latter shows the power law distribution equation. In drier conditions ( $-3 \mathrm{kPa}$ and higher), habitat fragmentation results in spatially isolated subpopulations growing at vastly different growth rates giving rise to the wide distribution in generation times. e) Fitted power law coefficient $\alpha$ for all hydration conditions. A lower coefficient indicates a more pronounced tail of the power law distribution (and suppression of the frequency of short generation times).

The study focuses on isogenic bacterial populations and their spatially distributed responses to conditions similar to a soil microbial hotspot and its surroundings where high nutrient concentrations support rapid cell proliferation near the nutrient source. The majority of realized generation times in a simulated hotspot are in the order of hours to days for most hydration conditions (shortest generation time at optimal conditions is $0.5 \mathrm{hr}$ ). However, the generation time distributions are characterized by persistence of very long generation times (a heavy tail), as shown in Fig. 3b. Interestingly, we find that under wet conditions (Fig. 3c), the tail of the distribution is truncated (i.e. fewer long generation times) due to bacterial motility through the water-saturated pore spaces that enable them to relocate towards more favorable conditions ${ }^{32}$. Under dry conditions (Fig. 3d), cell motility is greatly reduced and the dispersal range is restricted ${ }^{30}$ with reduced nutrient fluxes that result in a wide distribution of generation times following a power law (fitted power law and exponentially truncated power laws to the original and extrapolated simulation data are shown in Fig. $\mathrm{S} 1$ and S2, respectively). The fitted power law and exponentially truncated power law equations are shown in Equations 1 and 2 (with $\mathrm{x}$ the generation time, $\mathrm{x}_{\min }$ the minimum generation time of $0.5 \mathrm{~h}, \Gamma$ the gamma function, $\alpha$ the power law coefficient and $\lambda$ the exponential coefficient). 


\begin{tabular}{ll}
\hline$p(x)=(\alpha-1) * x_{\min }^{\alpha-1} * x^{-\alpha}$ & $(1)$ \\
\hline$p(x)=\frac{\lambda^{1-\alpha}}{\Gamma\left(1-\alpha, \lambda x_{\min }\right)} * x^{-\alpha} * e^{-\lambda x}$ & $(2)$ \\
\hline
\end{tabular}

The distributions of generation times in Fig. 3c, 3d, S3 and S4 were extrapolated by calculating the time required to complete a life cycle (generate the necessary biomass to cell division) based on observed growth rates. These estimated times are then added to the current cell age for predicting the most likely cell division under present and local conditions. We find that the main influence of hydration conditions lies in the suppression of rapidly dividing cells whilst simultaneously promoting the emergence of a more pronounced tail of the distribution (following diminishing nutrient conditions in drier conditions). The changes in the age distribution power law exponent with hydration status are summarized in Fig. 3e. The largest value of the exponent is associated with conditions under $-3 \mathrm{kPa}$ dominated by relatively short generation times. Under drier soil conditions, thin water films limit both bacterial motility ${ }^{30}$ and carbon diffusion ${ }^{6}$, resulting in slower growth and a more pronounced tail of the generation time distribution (lower $\alpha$ values in Fig. 3e). The choice of obligate aerobe as model bacterium implies sensitivity to saturated conditions with oxygen diffusion limitations irrespective of location, the resulting age distribution under these conditions must be interpreted with caution. The power law age distribution results for the unsaturated pore network (-3 to -7 $\mathrm{kPa}$ ) show a persistent decrease in the slope (lower values of $\alpha$ ) that reflect the disproportional suppression of the rapid growth in hotspots, with reduced effects on the already restricted growth in the cold spots that flatten the power law age distribution.

The resulting power law distribution of generation times in soil (as obtained from numerical simulations) can be linked more generally and analytically to microbial habitats that exhibit a wide distribution of cell growth rates ${ }^{24,33}$. A recent analysis showed that cell age distributions of an isogenic bacterial population growing in a chemostat follow an exponential decay ${ }^{33}$. Based on this assumption, the age distribution of a community consisting of many subpopulations growing at different rates is expected to follow a power law distribution (derivation in Appendix S1). An analogous derivation has been used for lag time distribution of cell rejuvenation leading to power law distributions of rejuvenation probabilities ${ }^{24}$. This analytical derivation is related to simulated conditions in dry soils, where immobilized cells and patchy nutrient conditions give rise to a wide distribution of growth rates as nutrient fluxes diminish or the aqueous phase becomes restrictive with soil drying (Fig. S3) leading to a power law age distribution for the respective population (Fig. S1 and S2).

Power law distributed cell ages induces a broad range of reproductive success where only few lineages dominate the total bacterial biomass in a community. Reproductive success is defined here as the maximum number of generations attributed to a lineage (parent cell) during the simulated time. Lineages with high reproductive success are those very few inoculated cells and their progeny that contribute disproportionally to the final population biomass, and are further defined as the dominant fraction. In contrast, a large proportion of the inoculated cells are incapable of proliferating and only contribute a minute fraction to the final biomass (rare fraction). To classify lineages into dominant and rare, we use a minimum cross-entropy algorithm originally developed for image thresholding ${ }^{34}$. Fig. 4a shows the fraction of the initial inoculum classified as either dominant or rare lineage. Interestingly, motility under wet conditions plays an important role as it enables individual cells to relocate towards favorable conditions, thereby equilibrating reproductive success between lineages rendering most of lineages as dominant. Under dry conditions, the combination of diffusion-limiting thin water films with pinning forces that limit cell dispersal ranges ${ }^{6,30}$ result in proliferation of only a few lineages that are close to the carbon source. More generally, conditions that support motion and migration equalize the contribution of lineages whereas restrictive conditions that limit dispersal and resources patchiness favor a few rare lineages. For all hydration conditions, the summed contribution of the rare lineages is less than $3 \%$ of the final population biomass. This stark difference in reproductive success between the rare and dominant lineages translates to equally prominent differences in their mean age distribution (Fig. 4b) and the mean generation times (Fig. 4c) of these lineages. The mean cell age of individual lineages diverges towards older cells for rare lineages and younger cells for dominant lineages with 
increasing matric potential (drier conditions) (Fig. 4b). The observed mean young cell age for rare lineages primarily stem from lineages that divided very late in the simulation due to growth close to the maintenance rate. Thus, these results suggest a bimodal distribution of bacterial age distributions in soil for bacterial cells growing close to a carbon source (hotspot) and within the bulk soil (cold spots) especially for drier conditions (Fig. S4). Similarly, there is a relationship between the average generation time and the relative abundance of individual lineages (Fig. 4c). Overall, rare lineages have a longer average generation time compared to dominant lineages, which is expected theoretically when considering the different reproductive success of the two groups.
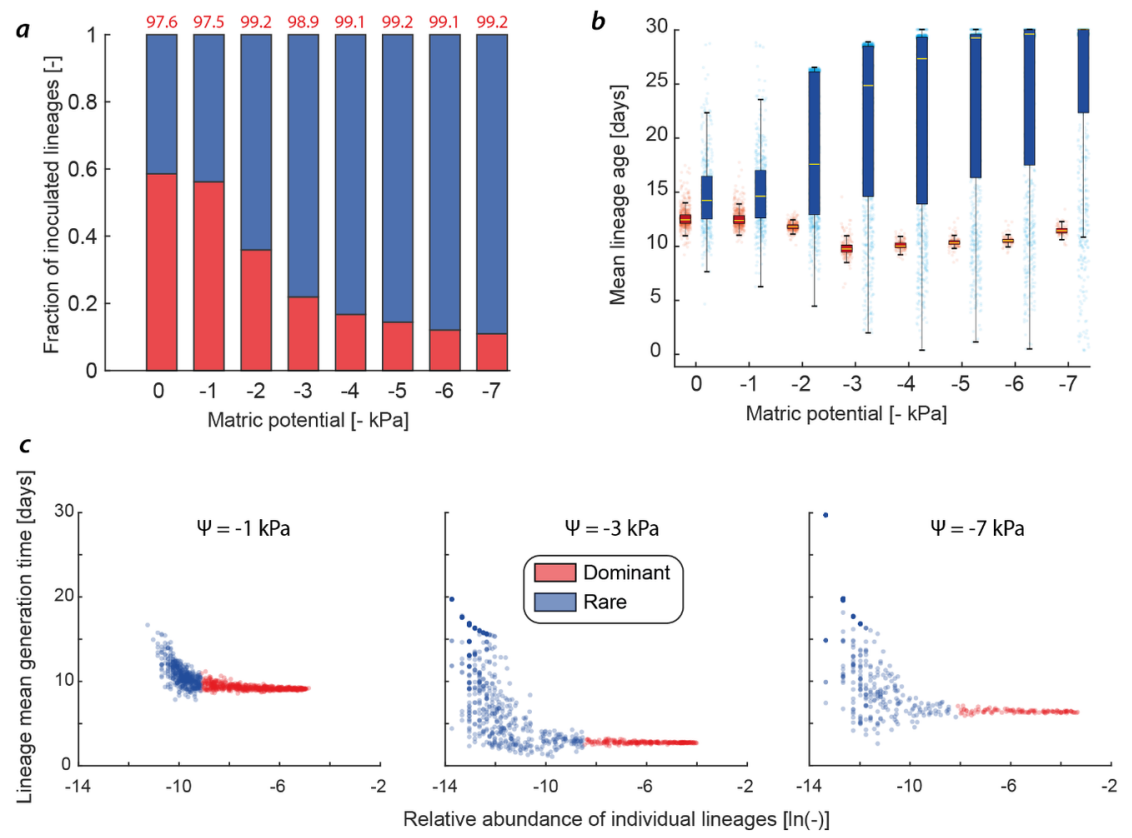

Figure 4: Characteristics of dominant (highest reproductive success) and rare lineages vary with hydration conditions. a) Percentage of lineages from the inoculum classified as dominant and rare. With a decline in bacterial motility towards drier conditions, most inoculated lineages cannot proliferate and contribute to the rare fraction of the community. Above numbers (in red) report the contribution (in percentage) of the dominant lineages to the final community biomass. b) Mean lineage cell age depending on their classification into rare and dominant shows an overall older cell population for the rare fraction due to their lower reproductive success. c) Mean lineage generation time in relation to their relative abundance $(\log )$ for three hydration conditions. The rare fraction is typically associated with longer mean generation times and only occurs in drier conditions where aqueous nutrient diffusion is limited to thin water films and capillary pinning forces immobilize bacterial cells.

\section{Discussion}

Access to patchy soil microenvironments is mediated by the (often fragmented) aqueous phase that controls diffusion of nutrients, activity of extracellular enzymes and the speed and range of bacterial cell dispersal. Nutrient rich domains disconnect from neighboring soil volumes shortly after episodic and short-lived wetting events thus maintaining broad distributions of growth rates and generation times of soil bacteria ${ }^{9,10}$. Limitations of the current experimental methods and inability to resolve soil bacterial age distributions motivated the use of mechanistic modeling that represents physical microhabitats and simulates individual bacterial cells interacting with their environments to track life histories of cell lineages and estimate generation time 
distributions. Simulation results show a broad range of growth rates marked by extremely slow growth rates similar to experimental observations in batch cultures where cells entered a deep starvation mode of balancing metabolic activity and cell maintenance (in line with the mechanism in the mechanistic simulations) in response to diminishing nutrient conditions ${ }^{35}$. Analogous observations have been made in fertile soils where average population doubling times exceeding 100 days are common and reproducible ${ }^{9}$, with experiments in harsher conditions suggesting that bacterial generation times may become indefinitely long as nutrient interception balances cell maintenance rates ${ }^{36-38}$.

Simulation results have consistently produced broad age distributions that follow a power law with varying slopes as a function of hydration conditions (Fig. 3, S1 and S2). A power law age distribution is also derived analytically based on heuristic assumptions (see derivation in Appendix S1). For both, the numerical and analytical models, the key ingredient for emergence of a power law bacterial age distribution is a wide range of growth rates within the system under consideration. This assumption is difficult to validate in situ, since current methods to determine growth rates in soil measure a sample average growth rate (which would bias the observed growth rate towards rapid growing cells) and cannot resolve the growth rate distribution of individual cells. The resulting wide distribution of growth rates in the mechanistic model is a consequence of the patchy nutrient landscape dictated by thin water films especially in dry conditions (Fig. S3) for which the simulation data show a generation time distribution following a power law more closely (Fig. $3 \mathrm{~d}$ ). Interestingly, the mechanistic model (that makes no assumptions regarding growth rate or population age distributions), and the simple analytical approach independently predict a power law distribution of soil bacterial generation time and cell age. A consequence of such distribution is that no simple mean bacterial generation time or cell age can be defined due to the heavy tail cell age distribution implying that a soil sample may harbor a nearly unbounded range of bacterial ages. We may define the average cell age of certain fractions of the population (Table S2), such estimates would however be highly biased towards the younger and more abundant cells. A deviation between the analytically derived power law coefficient $\alpha=2$ and fitted coefficients for numerical results in the range of 1.2 to 1.35 (Fig. 3e), suggesting that the mechanistic simulations predict a heavier tail of the distribution compared to analytical derivation. Given the higher level of complexity in the assumption-free mechanistic model, and inability to extract the real spectrum of cell growth rates and resource distribution, we defer resolution of the power law slope to future studies.

In addition to shaping cell growth rate distribution, hydration conditions affect population dynamics and induce a division of the bacterial community into dominant and rare lineages. By virtue of their position relative to nutrient sources, dominant lineages that contribute most to the overall biomass (but constitute the minority of the original inoculated cell lineages) proliferate and achieve high reproductive success, which translates to shorter generation times and younger cells on average. In contrast, the rare lineages grow very slowly, thereby exhibiting overall longer generation times and aged cells. Thus, considering a soil volume that contains at least a single hotspot, we expect the emergence of a bimodal distribution of cell ages with younger cells in the bacterial hotspots and old cells sparsely distributed in the bulk soil (cold spots) mediated by diffusional and dispersal constraints (Fig. S4). Although the present study focuses on an isogenic and copiotrophic bacterial species, our results show how the interplay of bacterial hot and cold spots and diffusion limitations provide mechanisms that support the emergence of within species diversity ${ }^{39}$. The mechanistic simulations suggest that even for isogenic cells, a large generational gap between different lineages emerges due to favorable spatial positioning, especially in dry conditions where motility is suppressed. In population genetic terms, the standing genetic variation in nascent soil bacterial hotspots, which is initially generated by mutations or gene flow, is primarily shaped by genetic drift as opposed to natural selection, facilitating the generation of within species diversity even at the smallest scale. In addition to promoting within species diversity, an interesting analogy exists between the rare and dominant lineages in our simulations and the rare and common species found in natural soil ${ }^{40}$. The key mechanism fostering a power law age distribution is a wide range of bacterial growth rates. Recent experimental results have provided glimpses into a wide distribution of taxon-specific growth rates for the same soil sample ${ }^{41}$, raising the question of whether bacterial cold spots (representing the rare lineages) also harbor the tail of the bacterial species distribution (rare 
species).

This discrepancy in dynamics between lineages (or strains/species) culminates in the creation of a "genetic reservoir" (also termed microbial genetic seed banks ${ }^{42}$ ) where rapid proliferation generates genetic variation that persists through time owing to the slow growing and rare lineages acting as keepers of traits. Often such genetic reservoirs have been associated with the fraction of the bacterial population at a state of reduced metabolic activity (dormancy) within larger soil volumes ${ }^{43}$. Our results suggest that even under ubiquitous hydrological conditions and within small soil-volumes $\left(\mathrm{mm}^{3}\right)$ occupied by a single bacterial hotspot, the persistence of rare lineages existing close to the maintenance rate provides a simple and intuitive mechanism for the emergence of heavy tailed generation time distributions that contribute to the soil bacterial genetic reservoir. Admittedly, the mechanistic model is a highly abstracted and simplified reality representing a small domain with limited heterogeneity where a single, aerobic species growing on a sole carbon source in absence of environmental variables (e.g. $\mathrm{pH}$ or temperature) with the exception of hydration conditions. Natural soil consists of numerous additional constraints such as highly complex pore spaces, restricted nutrient accessibility, a plethora of potential carbon sources and additional biotic factors (intraspecific variability, trophic interactions and non-growth associated maintenance). Since our simulations represent the optimal case of rapid bacterial growth, the above-mentioned processes are likely to extend the generation time distribution and contribute further to bacterial seed banks in soil ${ }^{43}$. In analogy, we also expect a range of biotic and abiotic processes to act in the opposite direction. These would truncate the range of applicability of power law bacterial age distribution. The lowest limit for the age range is defined by physiological constraints affecting rapid bacterial growth (i.e. the shortest biologically possible generation time), whereas mechanisms that truncate the upper range of the power law are more complicated to determine. Unlike animal age, the lifetime of a bacterial cell is not limited ${ }^{36-38}$. However, there are other mechanisms of bacterial death which shape the cell age distribution, such as grazing of bacteria ${ }^{44}$, large-scale bacterial death associated with episodic wetting events ${ }^{45}$ or phage infections ${ }^{46}$ supporting a "forever young" hypothesis ${ }^{47}$. If the mechanisms above act uniformly on the total population, they would affect primarily the dominant lineages (due to their high relative abundance), yet, over extended periods we expect gradual erosion of older cells and rejuvenation of the overall community. Since many of the above-mentioned mechanisms are related to the microscale liquid organization in soil pores, the resulting bacterial cell age and generation time distribution represents a delicate balance between processes that promote and suppress survival of old bacterial cells as a function of soil hydration conditions.

Both the extrapolated generation time calculation of the mechanistic model and the analytical derivation assume steady state conditions concerning bacterial growth rates. Steady state rarely exists in natural soil habitats, which are often subjected to diurnal and seasonal effects including episodic wetting events that reconnect the patchy environment and thus could homogenize conditions for a brief window of time. As a soil gradually dries, the brief homogenization of the conditions may result in reshuffling of lineages where previously proliferating lineages may experience harsh conditions and vice versa. Such resetting events have not been included in the mechanistic model nor in the analytical age model. In addition to shaping the bacterial age and generation time distributions, these stochastic events may facilitate horizontal gene transfer (especially in the subsequently drier conditions ${ }^{27}$ ) between genetically distant bacterial cells through reconnecting previously segregated patches and providing a mechanism to access the genetic reservoir directly.

The natural emergence of a soil genetic reservoir for each species (old bacterial cells inhabiting soil cold spots) may be specifically important for ecosystem stability and function. The dynamic nature of soil as a microbial habitat creates scenarios where adapted gene variants are outcompeted due to changing environmental conditions. If environmental conditions revert to a previous state, a genetic memory may promote rapid adaption of the soil microbial communities ${ }^{48}$. Evidence for the importance of the microbial seed bank in soil has been found in the disproportionate response of rare taxa during following rapid changes in environmental cues ${ }^{49}$ or during a controlled 45 -week experiment including a thermal disturbance ${ }^{50}$. In both cases, resuscitation of dormant taxa from the vast soil genetic reservoir was key for ecosystem stability and function. Additionally, the presence of old bacterial cells enhances the potential for horizontal gene transfer of ancient traits that may provide additional benefits. This is particularly true for soil where close 
proximity in dry soil (after an episodic wetting event that reconnected proximal soil volumes) increases the rate of horizontal gene transfer due to spatial confinement ${ }^{27}$.

The original aim of estimating bacterial cell age and generation time distributions in soils in relation to heterogeneity and hydration conditions began with a very simple question: "What is the average age of a soil bacterial cell?", and ended with an equally simple answer: "practically any age". In contrast to macrobiota with a finite life span where an average age in an ecosystem is well defined, providing a similar answer for prokaryotes living within heterogeneous soil is far more complicated. The proximity of vastly different ages and generations of the same bacterial species that may coexist a few hundred microns apart raise several intriguing possibilities. Considering reconnection of subpopulations with large generational gaps by episodic soil wetting events, could offer opportunities for regaining physiological traits lost during prolonged segregation and potentially provide a ubiquitous mechanism for sustaining genetic reservoir of traits and ecotypes ${ }^{39}$.

\section{Acknowledgments}

We thank Noah Fierer (University of Colorado, Boulder) for motivating this work with the question "what is the average age of soil bacteria? "We also thank Robin Tecon and Samuel Bickel (ETH, Zurich) for discussing the presented material on numerous occasions.

\section{References}

1. Bardgett, R. D. \& Van Der Putten, W. H. Belowground biodiversity and ecosystem functioning. Nature 505, 505-511 (2014).

2. Young, I. M. \& Crawford, J. W. Interactions and self-organization in the soil-microbe complex. Science 304, 1634-1637 (2004).

3. Kuzyakov, Y. \& Blagodatskaya, E. Microbial hotspots and hot moments in soil: Concept \& review. Soil Biol. Biochem. 83, 184-199 (2015).

4. Bååth, E. \& Johansson, T. Measurement of bacterial growth rates on the rhizoplane using 3H-thymidine incorporation into DNA. Plant Soil $126,133-139$ (1990).

5. Kieft, T. L. Size matters: Dwarf cells in soil and subsurface terrestrial environments. in Nonculturable Microorganisms in the Environment 19-46 (2000).

6. Tecon, R. \& Or, D. Biophysical processes supporting the diversity of microbial life in soil. FEMS Microbiol. Rev. $41,599-623$ (2017).

7. Van Heerden, J. H. et al. Statistics and simulation of growth of single bacterial cells: Illustrations with B. subtilis and E. coli.Sci. Rep. 7 , 1-11 (2017).

8. Clarholm, M. \& Rosswall, T. Biomass and turnover of bacteria in a forest soil and a peat. Soil Biol. Biochem. 12, 49-57 (1980).

9. Harris, D. \& Paul, E. A. Measurement of bacterial growth rates in soil. Appl. Soil Ecol. 1 , 277-290 (1994).

10. Rousk, J. \& Bååth, E. Growth of saprotrophic fungi and bacteria in soil. FEMS Microbiol. Ecol. 78 , 17-30 (2011).

11. Gibson, B., Wilson, D. J., Feil, E. \& Eyre-Walker, A. The distribution of bacterial doubling times in the wild. Proc. R. Soc. B Biol. Sci. 285, 20180789 (2018).

12. Schwartz, E. et al. Stable isotope probing with 18O-water to investigate microbial growth and death in environmental samples.Curr. Opin. Biotechnol. 41, 14-18 (2016). 
13. Purcell, A. M. et al. Quantitative stable isotope probing with $\mathrm{H} 218 \mathrm{O}$ to measure taxon-specific microbial growth. Methods Soil Anal. 4 , (2019).

14. Barber, D. A. \& Lynch, J. M. Microbial growth in the rhizosphere.Soil Biol. Biochem. 9, 305-308 (1977).

15. Söderberg, K. H. \& Bååth, E. Bacterial activity along a young barley root measured by the thymidine and leucine incorporation techniques. Soil Biol. Biochem. 30 , 1259-1268 (1998).

16. Olsson, S., Bååth, E. \& Söderström, B. Growth of Verticillium dahliae Kleb. hyphae and of bacteria along the roots of rape ( Brassica napus L.) seedlings. Can. J. Microbiol. 33 , 916-919 (1987).

17. Bowen, G. D. \& Rovira, A. D. Microbial colonization of plant roots. Annu. Rev. Phytopathol. 14, 121-144 (1976).

18. Zelenev, V. V., Van Bruggen, A. H. C. \& Semenov, A. M. 'BACWAVE,' a spatial-temporal model for traveling waves of bacterial populations in response to a moving carbon source in soil. Microb. Ecol.40, 260-272 (2000).

19. Bennett, R. A. \& Lynch, J. M. Bacterial growth and development in the rhizosphere ofgnotobiotic cereal plants. Microbiology125 , 95-102 (1981).

20. Christensen, H., Funck-Jensen, D. \& Kjøller, A. Growth rate of rhizosphere bacteria measured directly by the tritiated thymidine incorporation technique. Soil Biol. Biochem. 21 , 113-117 (1989).

21. Bloem, J., de Ruiter, P. C., Koopman, G. J., Lebbink, G. \& Brussaard, L. Microbial numbers and activity in dried and rewetted arable soil under integrated and conventional management. Soil Biol. Biochem. 27, 655-665 (1992).

22. Remus-Emsermann, M. N. P., Tecon, R., Kowalchuk, G. A. \& Leveau, J. H. J. Variation in local carrying capacity and the individual fate of bacterial colonizers in the phyllosphere. ISME J. $6,756-765$ (2012).

23. Borer, B., Ataman, M., Hatzimanikatis, V. \& Or, D. Modeling metabolic networks of individual bacterial agents in heterogeneous and dynamic soil habitats (IndiMeSH). PLOS Comput. Biol. 15, e1007127 (2019).

24. Şimşek, E. \& Kim, M. Power-law tail in lag time distribution underlies bacterial persistence. Proc. Natl. Acad. Sci. U. S. A.116, 17635-17640 (2019).

25. Borer, B., Tecon, R. \& Or, D. Spatial organization of bacterial populations in response to oxygen and carbon counter-gradients in pore networks. Nat. Commun. 9 , 1-11 (2018).

26. Kleyer, H., Tecon, R. \& Or, D. Rapid shifts in bacterial community assembly under static and dynamic hydration conditions in porous media.Appl. Environ. Microbiol. 86 , (2019).

27. Tecon, R., Ebrahimi, A., Kleyer, H., Levi, S. E. \& Or, D. Cell-to-cell bacterial interactions promoted by drier conditions on soil surfaces. Proc. Natl. Acad. Sci. U. S. A. 115, 9791-9796 (2018).

28. Dechesne, A., Wang, G., Gulez, G., Or, D. \& Smets, B. F. Hydration-controlled bacterial motility and dispersal on surfaces.Proc. Natl. Acad. Sci. 107, 14369-14372 (2010).

29. Ebrahimi, A. N. \& Or, D. Microbial dispersal in unsaturated porous media: Characteristics of motile bacterial cell motions in unsaturated angular pore networks. Water Resour. Res. 50 , 7406-7429 (2014).

30. Tecon, R. \& Or, D. Bacterial flagellar motility on hydrated rough surfaces controlled by aqueous film thickness and connectedness.Sci. Rep. 6 , 19409 (2016).

31. Ebrahimi, A. \& Or, D. Hydration and diffusion processes shape microbial community organization and function in model soil aggregates. Water Resour. Res. 51 , 9804-9827 (2015).

32. Lunn, M. et al. Quantifying the roles of immigration and chance in shaping prokaryote community structure. Environ. Microbiol. 8, 732-740 (2005). 
33. Painter, P. R. \& Marr, A. G. Mathematics of microbialpopulations.Annu. Rev. Microbiol. 22, 519-548 (1968).

34. Li, C. H. \& Lee, C. K. Minimum cross entropy thresholding.Pattern Recognit. 26 , 617-625 (1993).

35. Gray, D. A. et al. Extreme slow growth as alternative strategy to survive deep starvation in bacteria. Nat. Commun.10 , 1-12 (2019).

36. Greenblatt, C. L. et al. Micrococcus luteus - Survival in amber. Microb. Ecol. 48 , 120-128 (2004).

37. Johnson, S. S. et al. Ancient bacteria show evidence of DNA repair. Proc. Natl. Acad. Sci. U. S. A. $104,14401-14405$ (2007).

38. Jaakkola, S. T. et al. The complete genome of a viable archaeum isolated from 123-million-year-old rock salt. Environ. Microbiol. $18,565-579$ (2016).

39. Van Rossum, T., Ferretti, P., Maistrenko, O. M. \& Bork, P. Diversity within species: interpreting strains in microbiomes. Nat. Rev. Microbiol. 1-16 (2020).

40. Bickel, S. \& Or, D. Soil bacterial diversity mediated by microscale aqueous-phase processes across biomes. Nat. Commun. 11, 1-9 (2020).

41. Koch, B. J. et al. Estimating taxon-specific population dynamics in diverse microbial communities. Ecosphere 9, e02090 (2018).

42. Lennon, J. T. \& Jones, S. E. Microbial seed banks: The ecological and evolutionary implications of dormancy. Nat. Rev. Microbiol.9, 119-130 (2011).

43. Locey, K. J., Fisk, M. C. \& Lennon, J. T. Microscale insight into microbial seed banks. Front. Microbiol. 7, 2040 (2017).

44. Clarholm, M. Protozoan grazing of bacteria in soil-impact and importance. Microb. Ecol. 7, 343-350 (1981).

45. Blazewicz, S. J., Schwartz, E. \& Firestone, M. K. Growth and death of bacteria and fungi underlie rainfall-induced carbon dioxide pulses from seasonally dried soil. Ecology 95 , 1162-1172 (2014).

46. Shapiro, O. H., Kushmaro, A. \& Brenner, A. Bacteriophage predation regulates microbial abundance and diversity in a full-scale bioreactor treating industrial wastewater. ISME J. 4, 327-336 (2010).

47. Kuzyakov, Y. \& Mason-Jones, K. Viruses in soil: Nano-scale undead drivers of microbial life, biogeochemical turnover and ecosystem functions. Soil Biol. Biochem. 127, 305-317 (2018).

48. Overballe-Petersen, S. \& Willerslev, E. Horizontal transfer of short and degraded DNA has evolutionary implications for microbes and eukaryotic sexual reproduction. BioEssays 36 , 1005-1010 (2014).

49. Aanderud, Z. T., Jones, S. E., Fierer, N. \& Lennon, J. T. Resuscitation of the rare biosphere contributes to pulses of ecosystem activity. Front. Microbiol. 6 , 24 (2015).

50. Sorensen, J. W. \& Shade, A. Dormancy dynamics and dispersal contribute to soil microbiome resilience. Philos. Trans. R. Soc. B Biol. Sci. 375, 20190255 (2020). 